\title{
LIFETIME MEASUREMENTS OF HIGH POLARIZATION STRAINED- SUPERLATTICE GALLIUM ARSENIDE AT BEAM CURRENT >1 MILLIAMP USING A NEW 100kV LOAD LOCK PHOTOGUN*
}

\author{
J. Grames ${ }^{\#}$, P. Adderley, J. Brittian, J. Clark, J. Hansknecht, D. Machie, M.Poelker, M.L. Stutzman, \\ R. Suleiman, K. Surles-Law, Jefferson Laboratory, Newport News, VA 23606, U.S.A.
}

\begin{abstract}
A new GaAs DC high voltage load lock photogun has been constructed at Jefferson Laboratory (JLab), with improved vacuum and photocathode preparation capabilities. As reported previously, this gun was used to study photocathode lifetime with bulk GaAs at DC beam currents between 1 and $10 \mathrm{~mA}$. In this submission, lifetime measurements were performed using high polarization strained-superlattice GaAs photocathode material at beam currents to $1 \mathrm{~mA}$, with near bandgap light from a fiber based drive laser having picosecond optical pulses and RF time structure.
\end{abstract}

\section{INTRODUCTION}

Spin-polarized electron beams are produced at facilities worldwide using GaAs-based photocathodes inside DC high voltage photoguns. Specifically, spin-polarized electrons are produced by photoemission from semiconductor photocathodes using circularly polarized laser light with photon energy slightly larger than the semiconductor band gap. The vent/bake photoguns at CEBAF/JLab operate reliably with high polarization at average beam current $\sim 100 \mu \mathrm{A}$ [1] however a proposed high-profile parity-violation experiment, Qweak [2], requires prolonged operation at $\sim 300 \mu \mathrm{A}$. Future accelerators require even higher current, with electron ion collider designs starting at $1 \mathrm{~mA} \mathrm{[3-4]} \mathrm{and} \mathrm{extending} \mathrm{to}$ $250 \mathrm{~mA}$ [5]. To meet these challenges numerous technological advances are required, particularly in the fields of ultra-high vacuum (UHV), high voltage (HV), drive laser technology, photocathodes and beam handling. This work extends previous measurements performed at JLab designed to better appreciate the mechanisms that limit GaAs photogun operation at high average current [67]. Specifically, a high polarization strained-superlattice GaAs photocathode was illuminated with near-bandgap light at $780 \mathrm{~nm}$ from a fiber-based drive laser with RF structure, to produce $1 \mathrm{~mA}$ average continuous-wave (CW) beam current for an extended period of time. Details of this experiment are described below.

\footnotetext{
*Notice: Authored by Jefferson Science Associates, LLC under U.S. DOE Contract No. DE-AC05-06OR23177. The U.S. Government retains a non-exclusive, paid-up, irrevocable, world-wide license to publish or reproduce this manuscript for U.S. Government purposes. \#grames@jlab.org
}

\section{STATE OF THE ART PHOTOINJECTOR}

\section{High Polarization Photocathode}

Substantially more than half of the electromagnetic nuclear physics experiments conducted at JLab require highly polarized electron beams. To achieve polarization greater than $50 \%$ (i.e., theoretical limit of bulk GaAs), single strained-layer GaAs/GaAsP and strainedsuperlattice multi-layer GaAs/GaAsP photocathodes have been used. Of the two materials, strained-superlattice photocathodes provide higher polarization (Fig. 1) and greater $\mathrm{QE} \quad(\sim 1 \%$ versus $\sim 0.2 \%)$ at the wavelength corresponding to peak polarization. As a consequence, strained-superlattice GaAs is now the photocathode material of choice for the JLab nuclear physics program.

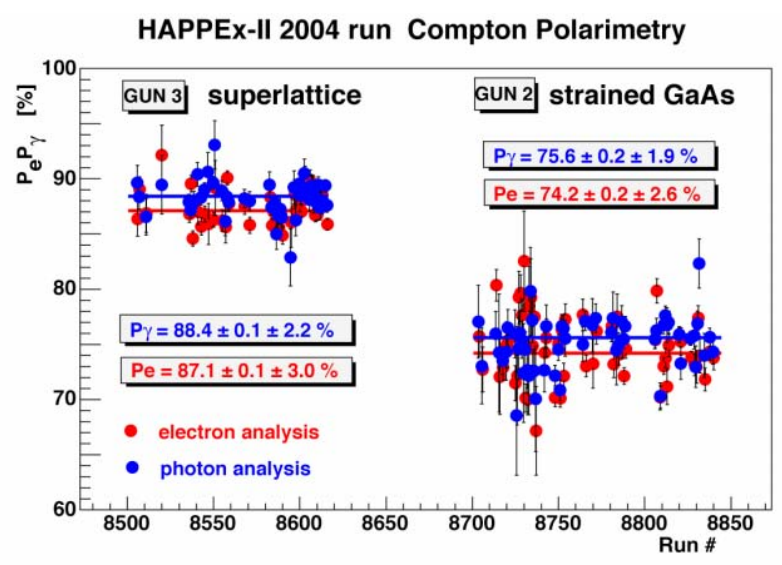

Figure 1: Electron polarization of strained-superlattice GaAs and single-layer strained GaAs (data from Hall A Compton polarimeter, courtesy JLab HAPPEx collaboration).

A strained-superlattice photocathode sample has been in near-constant use at CEBAF from September, 2005 through April, 2007, producing 1000 Coulombs of highly spin polarized electrons. During this period the photocathode was heated and activated seven times to restore QE. The electron polarization was found to be uniform across the activated area $(5 \mathrm{~mm}$ diameter $)$ and polarization remained constant over time despite photocathode damage via ion back-bombardment. Fig. 2 shows an example of constant polarization measured during a period when QE fell by a factor of two. 


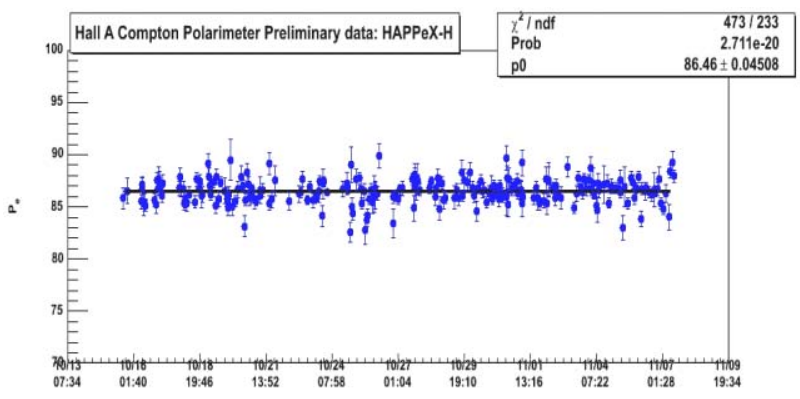

Figure 2: Electron polarization remains constant as photocathode QE drops by factor of two (data from Hall A Compton polarimeter, Courtesy JLab HAPPEx collaboration).

\section{Radio-Frequency Synchronous Fiber Laser}

A new laser ideally suited to driving strainedsuperlattice photocathodes and photoinjectors has been developed (Fig. 3) [8]. Light at $1560 \mathrm{~nm}$ from a gainswitched fiber-coupled diode laser and an ErYb-doped fiber amplifier is frequency doubled to obtain over $2 \mathrm{~W}$ average power at $780 \mathrm{~nm}$ with 40 ps pulses and pulse repetition rate of $499 \mathrm{MHz}$.

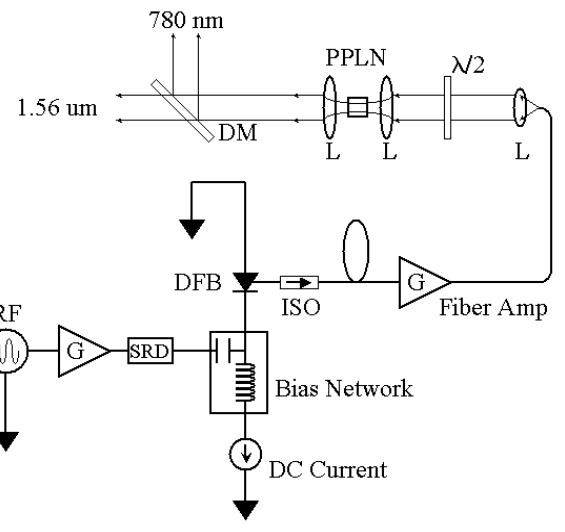

Figure 3: Schematic of the fiber-based laser system. DFB, distributed feedback Bragg reflector diode laser; ISO, fiber isolator; SRD, step recovery diode; L, lens; PPLN, periodically poled lithium niobate frequencydoubling crystal; DM, dichroic mirror.

\section{New Load Lock Electron Gun}

A new $100 \mathrm{kV}$ DC high voltage load lock photogun (Fig. 4) has been constructed, with improvements for photocathode preparation and ultra-high vacuum [7]. Although difficult to gauge directly, we believe the new gun has better vacuum compared to the previous gun [9], as evidenced by longer photocathode charge lifetime (i.e., the amount of charge extracted before QE falls to $1 / \mathrm{e}$ of the initial value).

The gun was commissioned with bulk GaAs and light at $532 \mathrm{~nm}$, and with different laser spot sizes (to be reported separately). The new gun exhibited exceptional performance at beam currents to $10 \mathrm{~mA}$, with charge lifetimes $>2000 \mathrm{C}$ using a $1.6 \mathrm{~mm}$ laser spot size and a charge density lifetimes $>10^{6} \mathrm{C} / \mathrm{cm}^{2}$ using a $0.32 \mathrm{~mm}$ diameter laser spot size [7].

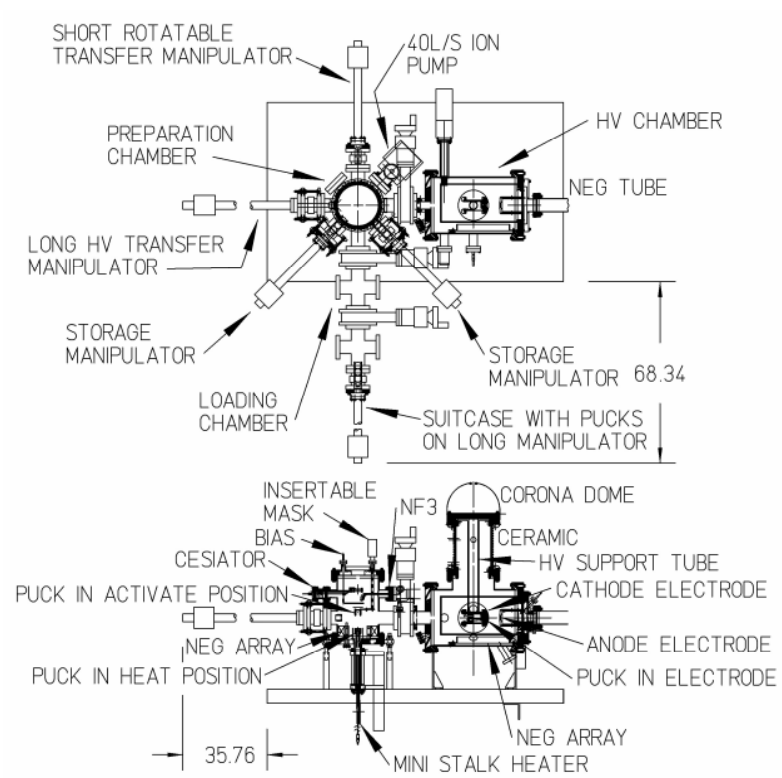

Figure 4: The new $100 \mathrm{kV}$ DC high voltage load lock photogun; with (top) plan view of the four vacuum chambers and manipulators and (bottom) side view of the preparation and $\mathrm{HV}$ chambers.

\section{EXPERIMENT}

The beam line used for photocathode lifetime measurements has been described previously [6-7] and is shown in Fig. 5. The electron beam exits the gun through a large bore ( 2.5 inch diameter) NEG-coated beam tube toward a 15 degree bend magnet. This bend allows illumination of the photocathode at normal incidence without using mirrors inside the vacuum chamber. Five solenoid magnets $(f \sim 50 \mathrm{~cm})$ and air core steering magnets were used to transport the beam $5.5 \mathrm{~m}$ to a Faraday cup that was previously degassed at $450^{\circ} \mathrm{C}$ for 24 hours to minimize outgassing and vacuum contamination at high current. In addition, two differential NEG pump stations provide beam line vacuum isolation from the Faraday Cup, a factor $>100$. Gun and beam line vacuum are monitored by high resolution $(<50 \mathrm{pA})$ ion pump power supplies. Viewers were used to verify proper beam transport through the center of all the solenoids and beam pipe. Magnetic shielding was added to uncovered beam pipe to dampen ambient DC and AC magnetic fields.

The fiber-based laser produced light at $780 \mathrm{~nm}$, the value for peak polarization, with 40 ps optical pulses and $499 \mathrm{MHz}$ pulse repetition rate (i.e., CEBAF conditions). The laser spot size was set by inserting a lens with appropriate focal length near the beam line vacuum window. The intensity profile of the laser beam was measured by diverting the beam, after the focusing lens, 


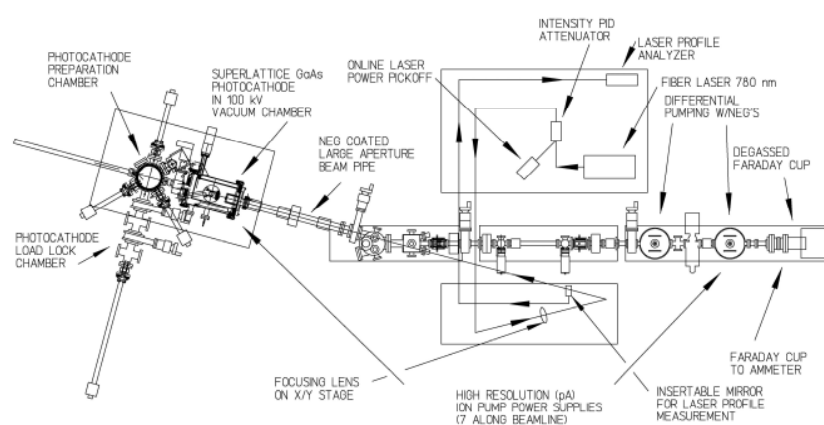

Figure 5: The new $100 \mathrm{kV}$ DC electron photogun, beam line and laser system is shown.

to a laser profile analyzer located at the photocathode distance. The laser intensity was remotely controlled using a polarization sensitive attenuator with high extinction ratio. This approach mitigated the need to use neutral density filters which enlarge and distort the laser profile. The laser power was occasionally measured using an in-line power meter inserted into the laser beam path, which interrupted beam delivery, or while running via a pick-off laser beam directed into a power meter calibrated to the in-line power meter.

A photocathode relative to the one in CEBAF providing the polarization data for Figs. 1-2 was used. The initial $\mathrm{QE}$ of the photocathode following activation was high, > $1 \%$. Lifetime measurements were made at beam currents of $250 \mu \mathrm{A}$ and $1 \mathrm{~mA}$ with a laser spot diameter of 0.45 $\mathrm{mm}$. A PID loop was used to automatically adjust the laser intensity attenuator to maintain constant current for each measurement. Before each measurement the vacuum conditions and initial QE across the photocathode active area were measured. During the run, beam current at the Faraday cup, pick-off laser power and relative vacuum pressure at six ion pump locations evenly distributed along the beam line were recorded. A beam current of 1 $\mathrm{mA}$ was demonstrated for 10 hours, extracting 30 Coulombs, with a $210 \mathrm{C}$ charge lifetime. A beam current of $250 \mathrm{uA}$ was demonstrated for 48 hours, extracting 70 Coulombs, with a charge lifetime of 250-1000 C. A false color image of the quantum efficiency following the lifetime measurements is shown in Fig. 6, where the "hole" near location $x=2600, y=2200$ demonstrates the ion back-bombardment is highly localized.

\section{CONCLUSIONS}

A high polarization strained superlattice GaAs photocathode was activated in the new load lock electron gun with $\mathrm{QE}$ of $\sim 1 \%$. This material was illuminated with light at $780 \mathrm{~nm}$ (wavelength for peak polarization) using an RF-pulsed fiber-based drive laser (40 ps pulses at 499 $\mathrm{MHz}$ ) to produce sustained $1 \mathrm{~mA}$ average continuous wave beam current. Photocathode charge lifetime was 210 Coulombs and higher at lower current. This test bodes well for the $\mathrm{Q}_{\text {weak }}$ experiment planned for Jefferson

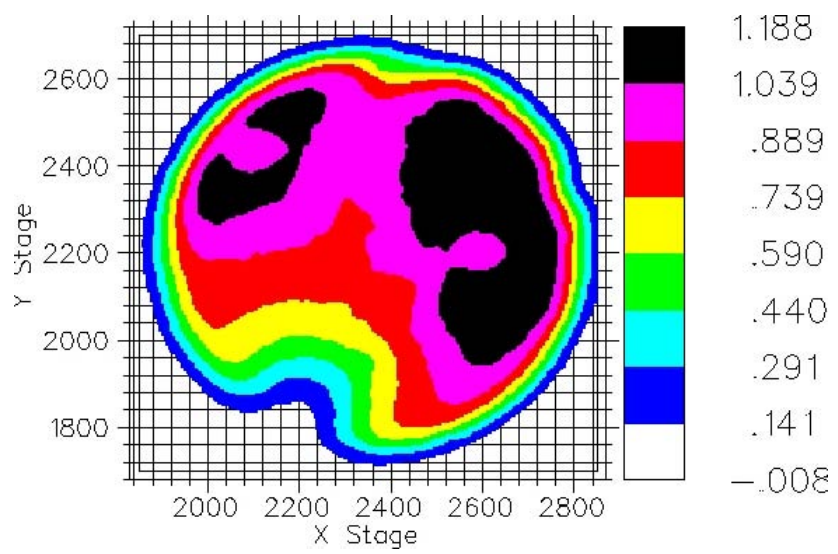

Figure 6: A false color plot of QE (in percent) following the $1 \mathrm{~mA}$ and $250 \mu \mathrm{A}$ runs, show QE reduction at the laser spot location (2600/2200). The portion of the photocathode in the lower, left quadrant had been damaged prior to these tests, by running beam under similar conditions.

Lab to begin in 2009, suggesting the new load lock photogun will meet increased high current demands. The test also demonstrates the feasibility of the Jefferson Lab EIC circulator ring proposal, to generate $>1 \mathrm{~mA}$ from a high polarization superlattice GaAs photocathode.

\section{REFERENCES}

[1] C. K. Sinclair et al., Phys. Rev. ST Accel. Beams 10, 023501 (2007).

[2] R. Carlini et al., "The $\mathrm{Q}_{\text {weak }}$ Experiment: A Search for New Physics at the TeV Scale", JLAB Experiment E02-020, December 6, 2004.

[3] Y. Derbenev et al., "Electron-Ion Collider at CEBAF: New Insights and Conceptual Progress" in Proceedings of the 2004 European Particle Accelerator Conference, Lucerne, Switzerland.

[4] L. Merminga et al., "ELIC: An Electron-Light Ion Collider based at CEBAF" in Proceedings of the 2002 European Particle Accelerator Conference, Paris, France.

[5] V. Ptitsyn et al., "eRHIC, Future Electron-Ion Collider at BNL" in Proceedings of the 2004 European Particle Accelerator Conference, Lucerne, Switzerland.

[6] M. Poelker and J. Grames, "Operation of CEBAF Photoguns at Average Beam Current $>1 \mathrm{~mA}$ " in Proceedings of the 2005 XIth International Workshop on Polarized Sources and Targets, Tokyo, Japan.

[7] J. Grames et al., "Further Measurements of Photocathode Operational Lifetime at Beam Intensity $>1 \mathrm{~mA}$ using the CEBAF $100 \mathrm{kV}$ DC GaAs Photogun", to appear in Proceedings of the 17 International Spin Symposium, Kyoto, Japan (2006).

[8] J. Hansknecht \& M. Poelker, Phys. Rev. ST Accel. Beams 9, 063501 (2006).

[9] W. Schneider et al., in Proceedings of the 1999 Particle Accelerator Conference, p. 1991. 